\title{
On the Single and Multiple Associations of COVID- 19 Post-acute Sequelae: 6-month Prospective Cohort Study
}

\section{Beatriz Maria Jiménez Rodríguez}

Hospital Universitario Virgen de las Nieves José Gutiérrez Fernández

Hospital Universitario Virgen de las Nieves

Eldis Maria Ramos Urbina

Hospital Universitario Virgen de las Nieves

Ana dolores Romero Ortiz

Hospital Universitario Virgen de las Nieves

\section{Paula Isabel García Flores}

Hospital Universitario Virgen de las Nieves

Maria Inmaculada Santiago Puertas

Hospital Universitario Virgen de las Nieves

\section{Maria Jose Martín López}

Hospital Universitario Virgen de las Nieves

Genaro López Milena

Hospital Universitario Virgen de las Nieves

Rene Fabregas ( $\nabla$ reneif07@gmail.com )

University of Manchester

Concepción Morales García

Hospital Universitario Virgen de las Nieves

\section{Research Article}

Keywords: Medical research, post-COVID characteristics, severe thromboembolic events, fatigue

Posted Date: August 12th, 2021

DOI: https://doi.org/10.21203/rs.3.rs-786903/v1

License: (c) (1) This work is licensed under a Creative Commons Attribution 4.0 International License.

Read Full License 


\section{Abstract}

Medical research is progressing to clarify the full spectrum of sub-acute and long-term effects of postacute sequelae of COVID-19. However, most manuscripts published to date only analyze the effects of long COVID in patients discharged from hospital, which may induce significant bias. Here, we propose a pioneering study to analyze the single and multiple associations between post-COVID characteristics with up to 6-months of follow-up in hospitalized and non-hospitalized covid-19 patients. The cohort study was conducted from May to October 2020 at the University Hospital Virgen de la Nieves, the leading hospital assigned for patients with COVID-19 in Granada, Spain. A total of 372 and 217 patients - with 217 and 207 included in the first and second follow-up visits - were referred 2 and 6 months after diagnosis of covid-19 infection, respectively. We find out that post-COVID-19 clinical and mental health impairment symptoms are correlated with patient gender. Logistic adjustments showed strong statistically robust single and multiple associations of demographic, clinical, mental health, X-ray, laboratory indices, and pulmonary function variables. The functional lung tests are good predictors of chest CT imaging abnormalities in elderly patients with long-haul COVID. Bilateral lung involvement, subpleural reticulum, ground-glass opacity, peripheral lung lesions, and bronchiectasis were the most common findings of the high-resolution computed tomography images. Non-hospitalized patients suffer more severe thromboembolic events and fatigue than those hospitalized.

\section{Introduction}

Long-term effects on multiple organ systems, caused by severe acute respiratory syndrome coronavirus 2 (SARS-CoV-2) - pathogen of coronavirus disease 2019 (COVID-19) - is one of the current problems faced by patients after passing the disease ${ }^{1-3}$. Preliminary studies report persisting symptoms of SARSCoV- 2 infections, such as fatigue, dyspnea, cognitive deficit, arthralgia, impaired lung functions, and abnormal chest images ${ }^{4-10}$. Similar persistent symptoms were reported in patients from previous coronavirus infections - including the 2003 SARS epidemic and the 2012 Middle East Respiratory Syndrome (MERS) - reinforcing concerns about post-acute sequelae of COVID-19 (PASC) ${ }^{11-16}$. The work of K.C. Ongsobre and colleagues clearly shows the persistent and prolonged effects of lung function impairment one year after acquiring severe acute respiratory syndrome (SARS1) ${ }^{15}$. In addition, Y.X. Liu and colleagues point out that deterioration of pulmonary functions and quality of life may occur up to 3 years after acute infection ${ }^{17}$.

Due to the limited capacity of the hospitals, only a tiny fraction of people with COVID-19 are admitted to hospitals ${ }^{18-21}$. However, most manuscripts published to date only consider pre-hospitalized patients for cohort studies, which may induce significant bias ${ }^{22}$. Current 12-, 6- and 3-month follow-up studies focus on persistent clinical, psychological, pulmonary function, physical problems, and chest CT imaging only for the discharged patients $8,10,23-28$. No study has yet reported on the scope of post-acute sequelae of COVID-19 in a singular or multiple manners, including the non-hospitalized patients. Also, the association between pre-existing respiratory diseases and PASC is still unknown. 
Our goal is to analyze the degree of single and multiple associations between clinical characteristics, demographic features, mental health, and pulmonary function test linked to PASC - of the first variant of SARS-CoV-2 - in patients with/without previous respiratory diseases, hospitalized or not, and the abnormalities of chest CT images.

\section{Results}

Of the 217 patients - of which 116 (53.5\%) were male - with SARS-CoV-2 infection was examined, including hospitalized and not hospitalized patients. These patients were monitored from May to October 2020. The follow-up study from May to October 2020 was divided into two follow-up consultations. The FFuC was carried out two months after the diagnosis of infection - from May to mid-July 2020 - and the SFuC six months after the initial diagnosis - from July to October 2020. A total of 148 patients were excluded from the study for the reasons set out in Fig. 1. The median and interquartile range for age and BMI were 59 (49-68) and 28 (26-32), respectively. Active smokers or ex-smokers 89 (41\%) with International Coalition Against Tobacco (ICAT) of $0[0-12,50]$. $52.6 \%$ had been in contact with family members with suspected or confirmed covid-19 infection. At the FFuC, the most prevalent symptoms were dyspnea in 138 (53.6\%) together with fatigue116 (53.5\%), emotional affectation $117(53.9 \%)$ and depression 124 (57.1\%). In 64 patients (30.3\%), the abnormal radiological findings continued. These and those with stress dyspnea were asked for chest HRCT. In the SFuC, 154 patients (73.3\%) still showed symptoms or claimed to develop new symptomatology after the acute process that was not attributable to alternative diagnoses. Dyspnea 88 (42.5\%), fatigue 99 (47.8\%), hair loss 47 (22.7\%), emotional affectation 91 (44\%), and depression 45 (21.7\%) were the most frequent symptoms. However, other alterations such as memory, concentration, and language deficits started to appear after the FFuC, reflecting a global cognitive deficit of up to $56(27.1 \%)$. They expressed it as a lack of mental fluency with stuttering and "brain fog" 29 . Also, erectile dysfunction or decreased sexual appetite was present in 3 $(1.4 \%)$ - not plotted. The overall results of the FFuC and SFuC are shown in Figure $\mathbf{S} 1$ of the supplementary material.

\section{Clinical outcomes and laboratory indices in female and males}

Figure 2 shows the clinical features, physical examination, and mental health of females/males with post-acute sequelae of SARS-CoV-2 infection - red affected and blue healthy - for first/second follow-up consultations. At the FFuC, the most frequent symptom - over $25 \%$ - in females [males] were dyspnea in 69 (68.3\%) [69 (40.5\%)], fatigue 61 (60.4\%) [55 (47.4\%)], emotional affectation 60 (59.4\%) [57 (49.1\%)], and depression 36 (35.6\%) [88 (75.9\%)], see Fig. 2A. Note here that in females, the symptoms of dyspnea, fatigue, and emotional affectation have a greater influence than in males, except for depression which affects more males than females. The symptoms of arthralgia, fever, and hair loss reject the chi-square null hypothesis of independence regarding gender - p-values less than $0.05-$ i.e., whether a person presents one of these symptoms is dependent on the person's gender. The rest of the less frequent 
symptoms - except epiphora - were still present after six months of the acute process. Figure $2 \mathrm{~B}$ shows the results for the SFuC where the significant features for females [males] were dyspnea 46 (48.4\%) [42 (37.5\%)], fatigue 58 (61.1\%) [41 (36.6\%)], hair loss 42 (44.2\%), emotional affectation 52 (54.7\%) [39 $(34.8 \%)]$, and depression $34(35.8 \%)$. All these symptoms are more frequent in females than in males. From the $\mathrm{p}$-values, we conclude that whether a person presents a lack of energy, emotional affectation, depression, or cognitive deficit depends on the person's gender. Also, decreased sexual appetite was observed in 1 female patient and erectile dysfunction in 2 males - not plotted.

The normal ( $\mathrm{N}$, green color) and abnormal (Abn., yellow color) laboratory indices - antibodies, hematologic, biochemical, infection and coagulation- are shown in Fig. 3 for females and males. At the FFuC - Fig. 3A -, a positive lgG and IgM were presented in 77 (80.2\%) [95 (90.5\%)] females [males] patients. This result confirms that both women and men have passed a relatively recent infection and are developing antibodies - as expected. A negative $\lg G$ and positive $\lg M$ or a positive $\lg G$ and negative $\lg M$ were presented in less than $25 \%$ of females and males. Also, Fig. $3 \mathrm{~A}$ and $3 \mathrm{C}$ show this cohort's most relevant abnormal indices in females [male] patients. Hemoglobin $34(31.8 \%)$ with 16 (15.7-16.4) - men only -, creatine $26(30.2 \%)$ with a median of $0.62(0.57-0.64)$, ferritin $25(32.1 \%)$ [73 (70.9\%)] with 160 (132-213) [259 (173-405)] and D-dimer 26 (38.2\%) [36 (38.3\%)] with $0.97(0.66-1.9)$ [0.83 (0.68-1.6)]. The indices of hemoglobin and serum ferritin reject the chi-square null hypothesis of independence regarding gender. The antibody test of the SFuC - Fig. 3B - shows that a positive IgG and IgM were presented in $44(48.9 \%)$ [47 (45.6\%)] females [males] patients. Also, 40 (44.4\%) [51 (49.5\%)] of females [males] were positive with $\lg$ and negative $\operatorname{lgM}$, e.i., the patients have been in contact with the virus and have generated antibodies after six months. Here all the frequency of abnormal laboratory indices for females is less than $25 \%$. However, more than one-quarter of male patients had abnormal hemoglobin [49 (47.6\%) with 16.2 (15.7-16.6)] and more than half abnormal ferritin [57 (57\%) with 198 (163-328)]. The indices of hemoglobin, serum ferritin - similar to the FFuC - , and the total bilirubin reject the chi-square null hypothesis of independence regarding gender. The median and the interquartile range for all the laboratory indices for females and males are represented in Fig. 3C. Here, the abnormal values of the indices for the different genera are clearly shown.

\section{Association between PASC features with pre-existing respiratory disease and hospitalization}

Figure 4 shows the odds ratio (OR) of PASC at 6-month follow-up using BVA and MVA, given the presence of a pre-existing respiratory disease (PRD) and hospitalization. A total of 207 patients were used for the BVA of gender, demographic characteristics, clinical features, mental health diseases, and hospitalization, of which 46 had the PRD. Also, we applied a BVA for lung exploration tests in a total of 157 patients, of which 33 had a PRD. For the MVA, we used 155 patients for all predictors, of which 33 had a pre-existing respiratory disease (see Fig. 4A). In addition, two hundred seven patients were used for the BVA with the same variables - except hospitalized - used for PRD analysis, of which 173 had been hospitalized. Also, for the BVA of the lung examination tests, 130 patients - of 157 - had been admitted to the hospital. The MVA included 33 patients - of 155 - that were discharged from the hospital (see Fig. 4B). The 
descriptive analysis, medians, and IQR of all the variables are shown in Figures $\mathbf{S} 2$ and $\mathbf{S} 3$ of the supplementary document.

After the bivariate adjustment, the following variables are in the robust (i.e., $2 \leq \mathrm{OR}$ ) range of ORs positively associated with a PRD compared to those without PRD: abnormal BMI, dyspnea, fatigue, emotional affectation, depression, impairment of FVC, FEV1, VR, DLCO, and FEV1/FVC - below their normal limits. The remaining features are in the range of less impressive $O R$ values (i.e., $0.5<O R<2$ ). Note that the highest OR corresponds to the FEV1/FVC ratio, implying that it has the highest bivalent association with the PRD compared to without PRD. Only the ORs of the abnormal BMI, dyspnea, fatigue, emotional affectation, depression, $\mathrm{FEV}<80 \%$, DLCO $<80 \%$, and $\mathrm{FEV} 1 / \mathrm{FVC}<70 \%$ ratio are statistically significant ( $p$-values $<0.05$ ). The ORs for abnormal BMI, ex-smokers, non-health workers, dyspnea, dry cough, loss of taste/smell, headache, emotional affectation, depression, all the impaired lung exploration tests, and the positive hospitalized status are in the positive or negative (i.e., $2 \leq \mathrm{OR}$ or $\mathrm{OR} \leq 0.5$ ) robust association range. The ORs of the remaining predictors are in a non-robust range. Statistically significant associations were found for abnormal BMI, ex-smokers, and dyspnea, as shown in Fig. 4A.

After the BVA, in the range of robust OR scores positive associated to hospitalized compared to nonhospitalized patients are the following features: male sex, age $>59$ years, active-smoker, ex-smokers, nonhealth worker, the impairment of FVC, FEV1, and 6MWT. Venous thrombosis is the only predictor with a negatively associated with hospitalized patients. The explanation for this result is that pharmacological thromboprophylaxis was provided during hospitalization. The remaining variables after the BVA are in the less impressive range of ORs, implying a negative association with hospitalized patients compared to non- hospitalized patients. Statistically significant associations were found for gender, age $>59$ years, non-health workers, and venous thrombosis (see Fig. 4B). After the MVA, the values of ORs for the male sex, non- health workers, age $<59$ years, fatigue, loss of taste/smell, headache, hair loss, venous thrombosis, depression, impairment of FEV1, KCO, and 6MWT are in the range of strongly associated scores to the hospitalization status. The remaining variables are in the interval of non-robust $\mathrm{OR}$ values. Predictors of males, non-health workers, fatigue, and venous thrombosis contribute significantly to the MVA (see Fig. 4b).

\section{Association between PASC features with chest high- resolution $\mathrm{CT}$ scan findings}

Figure 5A shows the chest high-resolution CT findings of 130 patients, of which 74 (56.9\%) presented chest image abnormalities due to consequences of COVID-19. The most notable - over $25 \%$ - features were: $68(52.3 \%)$ had bilateral pulmonary involvement, 37 (28.5\%) showed a subpleural reticular pattern, $56(43.1 \%)$ had a peripheral distribution, and 59 (45.4\%) exhibited a ground-glass opacity pattern. Less frequent CT findings were: unilateral lung involvement and interlobular reticular in 6 (4.6\%) patients, central (8 [6.2\%]) and central-peripheral $(7[5.4 \%])$ lesion location, mixed ground-glass opacity pattern (3 $[2.3 \%])$, bronchiectasis $(21[16.2 \%])$, emphysema (9 [6.9\%]), pulmonary thromboembolism (7 [5.4\%]), 
laminar atelectasis (2 [1.5\%]), and halo sign, pleural calcification, residual cavity, intrapulmonary node in 1 [0.8\%] patient (see Fig. 5a).

Figure 5B shows the odds ratio after bivariate and multivariate logistic adjustments to measure the association of gender, demographic characteristics, lung examination, pre-existing diseases, and hospitalization status with the abnormal CT outcome. A total of 109 patients - of 130 - were used for the BVA and MVA, of which 62 had abnormal CT results. The descriptive analysis, medians, and IQR of all the variables are shown in Figures $\mathbf{S 4}$ of the supplementary document. In bivariate analysis, males, age > 59 years, ex-smokers, abnormal radiography, impaired FEV1/FVC ratio, and a positive hospitalized status show a robust positive association (i.e., $\mathrm{OR}>2$ ). Non-smokers exhibit a robust negative $\mathrm{OR}$, and the remaining variables are in the range of less impressive OR scores. Male sex, age > 59 years, non-smoker, ex-smoker, and abnormal radiography are statistically significant. Here the relationship of each characteristic is calculated separately with the CT results. After MVA, the ORs for the male sex, age > 59 years, former smoker, non-smoker, abnormal radiograph, DLCO $<80 \%$, and FEV1/FVC $<70 \%$ in the robust interval of positively associated values. In addition, $\mathrm{FVC}<80 \%$ and pre-existing respiratory disease are robustly adversely correlated with an abnormal CT outcome. The ORs for the remaining characteristics are in the weak correlation range. Male sex, age > 59 years, and abnormal radiography were statistically significant.

\section{Discussion}

We have presented quantitative analyses demonstrating the presence of so-called post-acute sequelae in patients at 6-month follow-up. We started with a descriptive analysis of the clinical, mental health, physical examination, and laboratory indices findings. We have shown that the most prevalent characteristics are: dyspnea, fatigue, dry cough, loss of taste/smell, headache, hair loss, emotional affectation, depression, and cognitive deficit for clinical and mental health findings; and hemoglobin, lymphocytes, neutrophils, platelets, total bilirubin, creatine, ALT total protein, glucose, LDH, ferritin, and ddimer for abnormal laboratory indices. In addition, we have shown that fatigue, arthralgia, fever, hair loss, emotional affectation, depression, cognitive deficit, hemoglobin, total bilirubin, and ferritin associated with PASC depend on the patient's gender. This is in agreement with findings from previous long-term follow-up studies of SARS ${ }^{11,13}$ and MERS ${ }^{30,31}$.

At six months of follow-up, we found that the previous respiratory diseases and hospitalization status are strongly associated with specific demographic characteristics, clinical symptoms, mental health, and lung function tests based on single and multiple PASC features. In particular, the bivariate analysis demonstrated the robust statistically significant association of patients with previous respiratory diseases with the following essential features: abnormal BMI, as a demographic characteristic, dyspnea and fatigue as clinical features, emotional affectation and depression as psychological complications, and impaired of forced expiratory volume in one second, pulmonary diffusion capacities, and the FEV1/FVC ratio (i.e., positive diagnostic of obstructive and restrictive lung disease). However, after using more than one independent PASC feature, only the abnormal BMI, ex-smoker, and dyspnea had a robust 
statistically significant association to patients with previous respiratory diseases. This result implies that patients with pre-existing respiratory diseases with one or more PASC features need to be monitored on a long-term follow-up basis. The following characteristics were obtained from the bivariate analysis for hospitalization status with a robust association and significant relationships: male sex, older than 59 years old, non-health worker, and venous thrombosis. Nevertheless, after the multivariate analysis, the robustness of the association for the predictors increased - except for the age $>59$ years - , adding fatigue as a new statistically significant characteristic. The influential negative association of thromboembolic events $-\mathrm{OR}<<1-$ and fatigue suggests that patients without hospitalization also need long-term follow-up.

We found that the most common abnormal CT patterns were bilateral lung involvement, subpleural reticular, ground-glass opacity density with a peripheral location, which agrees with the previous results $8,10,25,32$. Our study revealed a significant robust positive single correlation between the abnormal CT findings and male sex, age $>59$ years, ex-smoker, and abnormal radiography, as well as multiple correlations, except for ex-smoker. Abnormal CT findings are strongly associated with the affected FVC, DLCO, and FEV1/FVC, yet, it is not statistically significant.

The major strength of our study is the long-term follow-up of patients with the examination of all patients reported at 2 and 6 months, including hospitalized and non-hospitalized patients. This study is the first to present single and multiple characterizations of the long-term sequelae of COVID-19. Moreover, our study is, to date, one of the most detailed and most prolonged follow-up studies of post-COVID-19 patients. However, the greater willingness of symptomatic patients to participate in a follow-up study is a possible biasing factor - as in all observational studies. The study findings may be limited due to the single-center study, nonblinded, and nonrandomized design. We understand this potential localization bias.

\section{Conclusions}

At six months follow-up, PASC characteristics fatigue, arthralgia, fever, breathlessness, emotional disturbance, depression, cognitive deficit, hemoglobin, total bilirubin, and ferritin are correlated with the gender of the patient. Patients with previous respiratory diseases and abnormal body mass index, exsmoker, and dyspnea had a robust statistically significant association. Non-hospitalized patients may suffer more severe thromboembolic events and fatigue than hospitalized patients. Functional lung tests are good predictors of chest CT imaging abnormalities in elderly patients with PASC.

The preliminary study presented here can be extended in a number of ways. First, the study can be prolonged to 12, 24, and 36 months of follow-up. This will enable us to study the long-term effects of post-COVID-19 symptoms and define different degrees of severity. Second, adding new variables to the study will allow us to create models to predict the most frequent symptoms for medical treatments. In fact, we are working to improve our study in these directions.

\section{Materials And Methods}




\section{Study population}

The prospective cohort study was conducted at the University Hospital Virgen de la Nieves, one of the hospitals assigned for patients with COVID-19 in Granada, Spain. Two visits were scheduled in the followup period from May to October 2020. A total of 372 patients - with 217 included and 115 excluded were referred 2-months after diagnosis of covid-19 infection - PCR or serology confirmed - for the first follow-up consultation (FFuC). The 217 patients included in the FFuC were referred to the second followup consultation (SFuC) at 6-months after initial diagnosis- with 207 included and 10 excluded. The study timeline, flowchart, and follow-up consultation procedures are shown in Fig. 1. The study was conducted following the requirements of the Declaration of Helsinki and the Spanish Data Protection Act of 15/1999. Following the Declaration of Helsinki, written informed consent was obtained from all patients, and local ethics committees approved the study.

The study population consisted of patients aged $>14$ years, diagnosis of infection confirmed according to international recommendations and signing the informed consent. The exclusion criteria were: suspected cases of SARS-CoV-2 infection, immunosuppressed patients with therapeutic limitation due to terminal pathology, and those who refused to participate (see Fig. 1). The age, sex, BMI, toxic habits, profession, family members with confirmed or suspected covid- 19 infection, and need or not of hospital admission were considered. See Table 2 of the supplementary material, which summarizes the characteristics of the patients included in the study.

\section{Data collected in follow-up consultations}

The clinical features studied at each follow-up visit were: dry and wet cough, dyspnea, fatigue, muscle weakness, musculoskeletal involvement, chest pain, palpitations, fever, sweating, intestinal disorders, post covid arterial hypertension, otorhinolaryngologic symptoms, ocular symptoms, neurological manifestations, decreased sexual appetite, cutaneous manifestations and other expressed symptoms thromboembolic events. Also, each consultation included mental abnormalities, physical examination, and laboratory indices (see Tables 1 and 2 of the supplementary material).

A chest radiograph was performed in posterior-anterior and lateral projection at the FFuC in all patients. Then, a complementary study with HRCT was requested if there were any abnormal findings on the X-ray. HRCTs were evaluated by radiologist specialists and one pulmonologist and reported according to the Spanish Society of Medical Radiology (SERAM) recommendations, the international standard nomenclature defined by the Fleischner Society glossary and existing publications until now. Each imaging test was analyzed considering: density (ground glass, consolidation or mixed), lung involvement (unilateral or bilateral), location (central, peripheral or mixed), presence of reticular pattern or interstitial lesions of pulmonary parenchymal (subpleural or interlobular), and the percentage of lung extension involved $<20 \%, 20-50 \%$ and $>50 \%$ - according to the lung fields involved (see Table 2 of the supplementary material). 
At the SFuC, each patient underwent forced spirometry, lung volume, diffuse capacity, and the 6-minute walk test (TM6M). The functional exploration was carried out with experienced personnel with the equipment of MasterScreen Body, brand Jaeger, Germany. The reference values for the Mediterranean population and acceptability criteria according to European and Spanish regulations. Pulmonary parameters included forced expiratory volume in the first second (FEV1), forced vital capacity (FVC), the FEV1/FVC ratio, pulmonary resistance, residual volume (RV), residual functional capacity (RFC), total lung capacity (TLC), carbon monoxide transfer by single breath (DLCO) and diffusion constant for carbon monoxide (KCO).

\section{Statistical analysis}

Descriptive analysis was carried out using number (\%), median, and its interquartile range (IQR) combining box plots and density plots, i.e., violin plots - for categorical and continuous variables, respectively ${ }^{33,34}$. Discrepancies in the patient characteristic distributions by sub-groups of outcomes are presented as differences with $95 \%$ confidence intervals (Cls). The Mann-Whitney U test - for nonnormal distributed continuous data,$- \chi^{2}$ test, or Fisher's exact was used to compare clinical features, physical examination, mental health, and laboratory indices between males and females at the first and second follow-up consultations.

Bivariate and multivariate analysis was carried out to compute the odds ratios (ORs) and $95 \%$ Cls to explore the association with the following features: at least one pre-existing respiratory disease, hospitalized patients, and an abnormal chest CT finding ${ }^{35}$. The degree of association of the long-COVID symptoms is defined according to the $\mathrm{OR}$ value (robust or not). Data cleaning and analysis using logistic regression models were implemented in Python 3.7. The tests were two-sided, and a p-value less than $a=$ 0.05 was considered statistically significant.

\section{List Of Abbreviations That Were Used}




\begin{tabular}{|ll|}
\hline SARS-CoV-2 & Severe acute respiratory syndrome coronavirus 2 \\
\hline SARS-1 & Severe acute respiratory syndrome \\
\hline MERS & Middle East Respiratory Syndrome \\
\hline PASC & Post-acute sequelae of CoVID-19 \\
\hline ICAT & Coalition Against Tobacco \\
\hline BMI & Body mass index \\
\hline FFuC & First follow-up consultation \\
\hline SFuC & Second follow-up consultation \\
\hline X-Ray & Chest radiograph \\
\hline CT & Chest computed tomography \\
\hline HRCT & High-resolution computed tomography \\
\hline GGO & Ground glass opacity pattern \\
\hline FEV1 & Forced expiratory volume in the first second \\
\hline FVC & Forced vital capacity \\
\hline FEV1/FVC & The FEV1/FVC ratio \\
\hline RV & Residual volume \\
\hline FRC & Residual functional capacity \\
\hline TLC & Total lung capacity \\
\hline DLCO & Carbon monoxide transfer by a single breath \\
\hline KCO & Diffusion constant for carbon monoxide \\
\hline TM6M & The 6-minute walk test \\
\hline SERAM & Spanish Society of Medical Radiology \\
\hline IQR & Interquartile range \\
\hline Cls & Confidence intervals \\
\hline ORs & Odds ratios \\
\hline BVA & Biviate analysis \\
\hline MVA & Priate analysis \\
\hline
\end{tabular}




\section{Declarations}

\section{Author contributions}

C.M.N. proposed and directed the research with help from B.M.J.R., J.G.F., and R.F.; M.I.S.P., and M.J.M.L carried out the measurement and data collection of the pulmonary function tests; B.M.J.R. collected the demographic, clinical, and psychological data in follow-up consultations supported by P.I.G.F. and E.M.R.U.; G.L.M. and B.M.J.R. performed the acquisition and processing of HRCT images; J.G.F. undertook the processing of the laboratory indices; R.F. and B.M.J.R conducted the data analysis in Python; C.M.N, B.M.J.R., J.G.F., and R.F. wrote the manuscript; and all authors contributed to discussions.

\section{Acknowledgments}

The ethical approval of this prospective cohort study was granted by the Ethics Committee of the Hospital Universitario Virgen de las Nieves. This research work is associated with the doctoral thesis at the University of Granada of Beatriz María Jiménez Rodríguez.

\section{Declaration of interests}

The authors declared no conflict of interest.

\section{Data sharing}

Currently, the third follow-up consultation (one year after the disease) is being collected, which means that data cannot be shared. Once the data analysis process for each follow-up process is completed, the data and the implemented code could be shared on an internet hosting, such as GitHub repository.

\section{References}

1 Mokhtari, T. et al. COVID-19 and multiorgan failure: A narrative review on potential mechanisms. J Mol Histo/ 51, 613-628, doi:10.1007/s10735-020-09915-3 (2020).

2 Gupta, A. et al. Extrapulmonary manifestations of COVID-19. Nat Med 26, 1017-1032, doi:10.1038/s41591-020-0968-3 (2020).

3 Nalbandian, A. et al. Post-acute COVID-19 syndrome. Nature medicine, 1-15, doi:10.1038/s41591-021-01283-z (2021).

4 Carfi, A., Bernabei, R., Landi, F. \& Group, f. t. G. A. C.-P.-A. C. S. Persistent Symptoms in Patients After Acute COVID-19. JAMA 324, 603-605, doi:10.1001/jama.2020.12603 (2020).

$5 \quad$ Xiong, Q. et al. Clinical sequelae of COVID-19 survivors in Wuhan, China: a single-centre longitudinal study. Clin Microbiol Infect 27, 89-95, doi:10.1016/j.cmi.2020.09.023 (2021). 
Huang, Y. et al. Impact of coronavirus disease 2019 on pulmonary function in early convalescence phase. Respiratory Research 21, 163, doi:10.1186/s12931-020-01429-6 (2020).

$7 \quad$ Zhao, Y.-M. et al. Follow-up study of the pulmonary function and related physiological characteristics of COVID-19 survivors three months after recovery. doi:10.1016/j.eclinm.2020.100463 (2020).

8 Xiong, Y. et al. Clinical and High-Resolution CT Features of the COVID-19 Infection: Comparison of the Initial and Follow-up Changes. Investigative Radiology 55, 332-339, doi:10.1097/RLI.0000000000000674 (2020).

9 Tenforde, M. W. et al. Symptom Duration and Risk Factors for Delayed Return to Usual Health Among Outpatients with COVID-19 in a Multistate Health Care Systems Network - United States, MarchJune 2020. MMWR Morb Mortal Wkly Rep 69, 993-998, doi:10.15585/mmwr.mm6930e1 (2020).

10 Huang, C. et al. 6-month consequences of COVID-19 in patients discharged from hospital: a cohort study. The Lancet 397, 220-232, doi:10.1016/S0140-6736(20)32656-8 (2021).

11 Ong, K. C. et al. 1-Year pulmonary function and health status in survivors of severe acute respiratory syndrome. Chest 128, 1393-1400, doi:10.1378/chest.128.3.1393 (2005).

12 Hui, D. S. et al. Impact of severe acute respiratory syndrome (SARS) on pulmonary function, functional capacity and quality of life in a cohort of survivors. Thorax 60, 401-409, doi:10.1136/thx.2004.030205 (2005).

13 Lam, M. H. et al. Mental morbidities and chronic fatigue in severe acute respiratory syndrome survivors: long-term follow-up. Arch Intern Med 169, 2142-2147, doi:10.1001/archinternmed.2009.384 (2009).

14 Moldofsky, H. \& Patcai, J. Chronic widespread musculoskeletal pain, fatigue, depression and disordered sleep in chronic post-SARS syndrome; a case-controlled study. BMC Neurol 11, 37, doi:10.1186/1471-2377-11-37 (2011).

15 Ong, K. C. et al. Pulmonary function and exercise capacity in survivors of severe acute respiratory syndrome. Eur Respir J 24, 436-442, doi:10.1183/09031936.04.00007104 (2004).

16 Lee, A. M. et al. Stress and psychological distress among SARS survivors 1 year after the outbreak. Can J Psychiatry 52, 233-240, doi:10.1177/070674370705200405 (2007).

17 Liu, Y. X. et al. Changes in pulmonary function in SARS patients during the three-year convalescent period. Zhongguo Wei Zhong Bing Ji Jiu Yi Xue 19, 536-538 (2007).

18 Sen-Crowe, B., Sutherland, M., McKenney, M. \& Elkbuli, A. A Closer Look Into Global Hospital Beds Capacity and Resource Shortages During the COVID-19 Pandemic. Journal of Surgical Research 260, 56- 
63, doi:https://doi.org/10.1016/j.jss.2020.11.062 (2021).

19 Shoukat, A. et al. Projecting demand for critical care beds during COVID-19 outbreaks in Canada. Canadian Medical Association Journal 192, E489, doi:10.1503/cmaj.200457 (2020).

$20 \mathrm{Li}$, R. et al. Estimated Demand for US Hospital Inpatient and Intensive Care Unit Beds for Patients With COVID-19 Based on Comparisons With Wuhan and Guangzhou, China. JAMA Network Open 3, e208297-e208297, doi:10.1001/jamanetworkopen.2020.8297 (2020).

21 Barrett, K. et al. Estimation of COVID-19-induced depletion of hospital resources in Ontario, Canada. Canadian Medical Association Journal 192, E640, doi:10.1503/cmaj.200715 (2020).

22 Griffith, G. J. et al. (2020).

23 Seeßle, J. et al. Persistent symptoms in adult patients one year after COVID-19: a prospective cohort study. Clinical Infectious Diseases, doi:10.1093/cid/ciab611 (2021).

24 Zhao, Y.-m. et al. Follow-up study of the pulmonary function and related physiological characteristics of COVID-19 survivors three months after recovery. EClinicalMedicine 25, 100463, doi:https://doi.org/10.1016/j.eclinm.2020.100463 (2020).

25 González, J. et al. Pulmonary Function and Radiologic Features in Survivors of Critical COVID19: A 3-Month Prospective Cohort. Chest, doi:10.1016/j.chest.2021.02.062 (2021).

26 Rass, V. et al. Neurological outcome and quality of life three months after COVID-19: a prospective observational cohort study. European Journal of Neurology, ene.14803-ene.14803, doi:10.1111/ene.14803 (2021).

27 Guan, W.-j. et al. Clinical Characteristics of Coronavirus Disease 2019 in China. New England Journal of Medicine 382, 1708-1720, doi:10.1056/nejmoa2002032 (2020).

28 Arentz, M. et al. Vol. 323 1612-1614 (American Medical Association, 2020).

29 Shah, W., Hillman, T., Playford, E. D. \& Hishmeh, L. Managing the long term effects of covid-19: Summary of NICE, SIGN, and RCGP rapid guideline. The BMJ 372, doi:10.1136/bmj.n136 (2021).

30 Ahmed, H. et al. Long-term clinical outcomes in survivors of severe acute respiratory syndrome and Middle East respiratory syndrome coronavirus outbreaks after hospitalisation or ICU admission: A systematic review and meta-analysis. J Rehabil Med 52, jrm00063, doi:10.2340/16501977-2694 (2020).

31 Lee, S. H. et al. Depression as a Mediator of Chronic Fatigue and Post-Traumatic Stress Symptoms in Middle East Respiratory Syndrome Survivors. Psychiatry Investig 16, 59-64, doi:10.30773/pi.2018.10.22.3 (2019). 
32 Xie, L. et al. Follow-up Study on Pulmonary Function and Lung Radiographic Changes in Rehabilitating Severe Acute Respiratory Syndrome Patients After Discharge. Chest 127, 2119-2124, doi:https://doi.org/10.1378/chest.127.6.2119 (2005).

33 Cleveland, W. S. \& McGill, R. Graphical Perception and Graphical Methods for Analyzing Scientific Data. Science 229, 828-833, doi:10.1126/science.229.4716.828 (1985).

34 Hintze, J. L. \& Nelson, R. D. Violin Plots: A Box Plot-Density Trace Synergism. The American Statistician 52, 181-184, doi:10.1080/00031305.1998.10480559 (1998).

35 Denis, D. J. Applied Univariate, Bivariate, and Multivariate Statistics Using Python: A Beginner's Guide to Advanced Data Analysis. (Wiley, 2021).

\section{Figures}




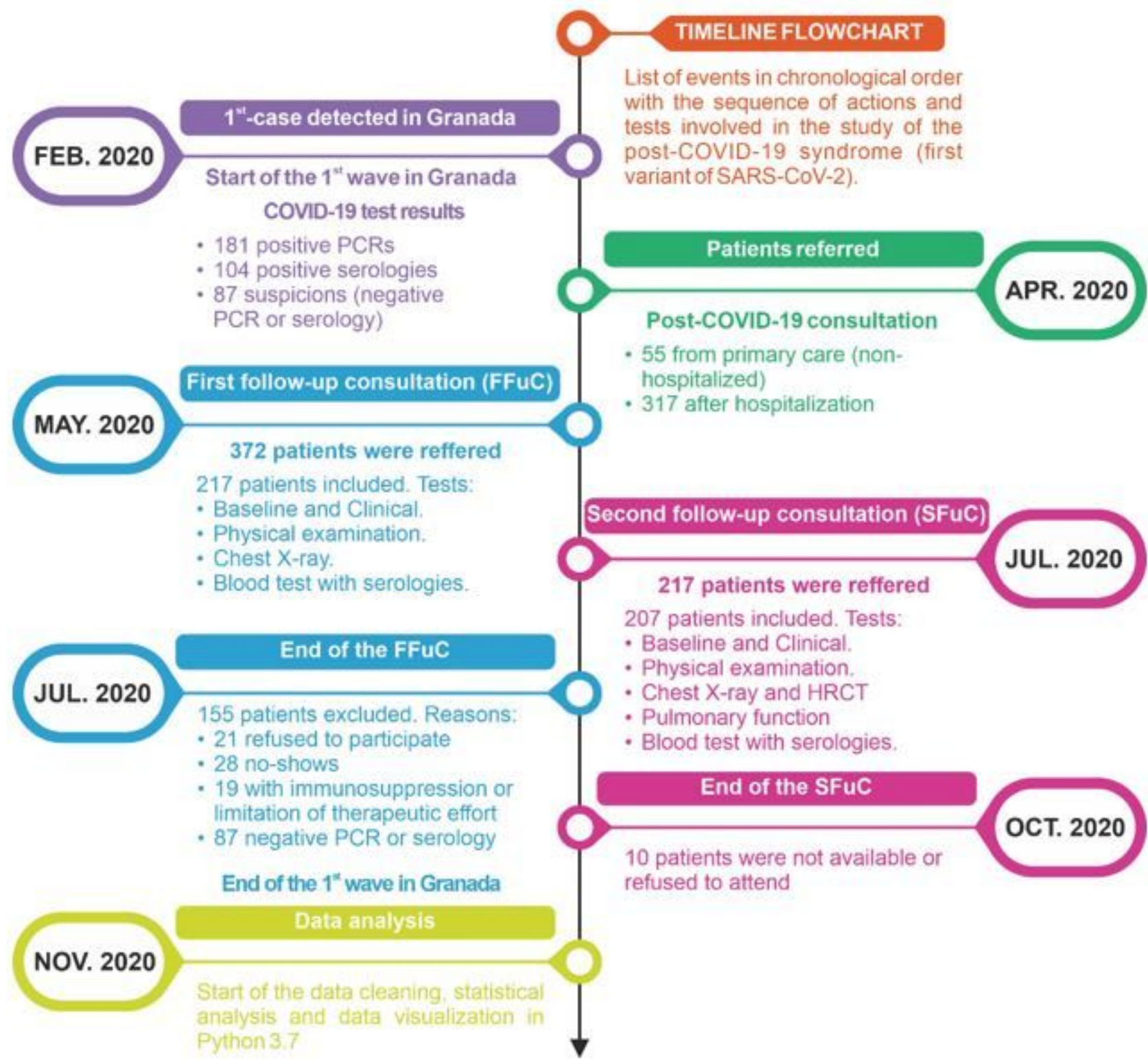

\section{Figure 1}

Timeline and flowchart illustrating the series of events in chronological order with the actions and tests conducted after the first case detected at the University Hospital Virgen de las Nieves. Starting with the first Covid-19 test results, the number of patients referred to post Covid-19 consultations, the details of the first and second consultations, and data analysis. 
A

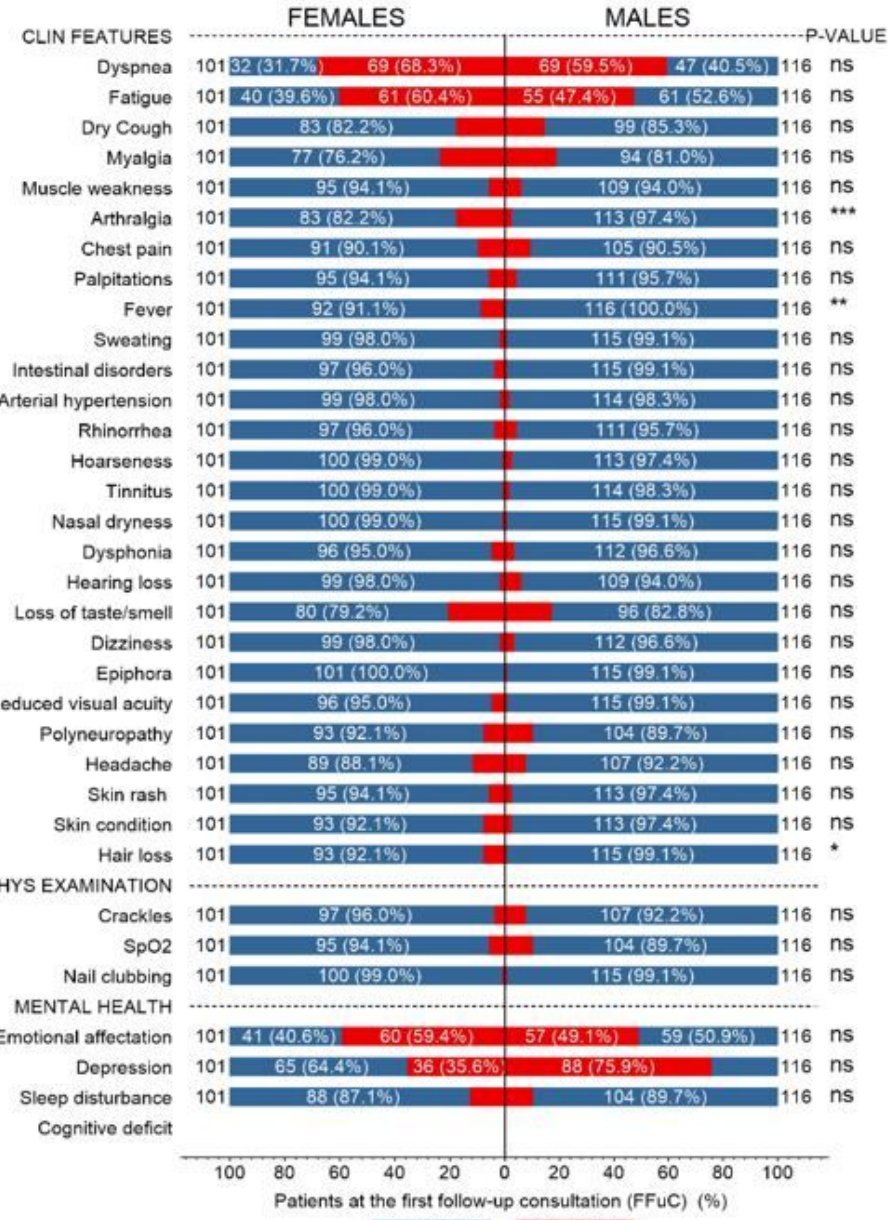

Hearisy EAfrected
B

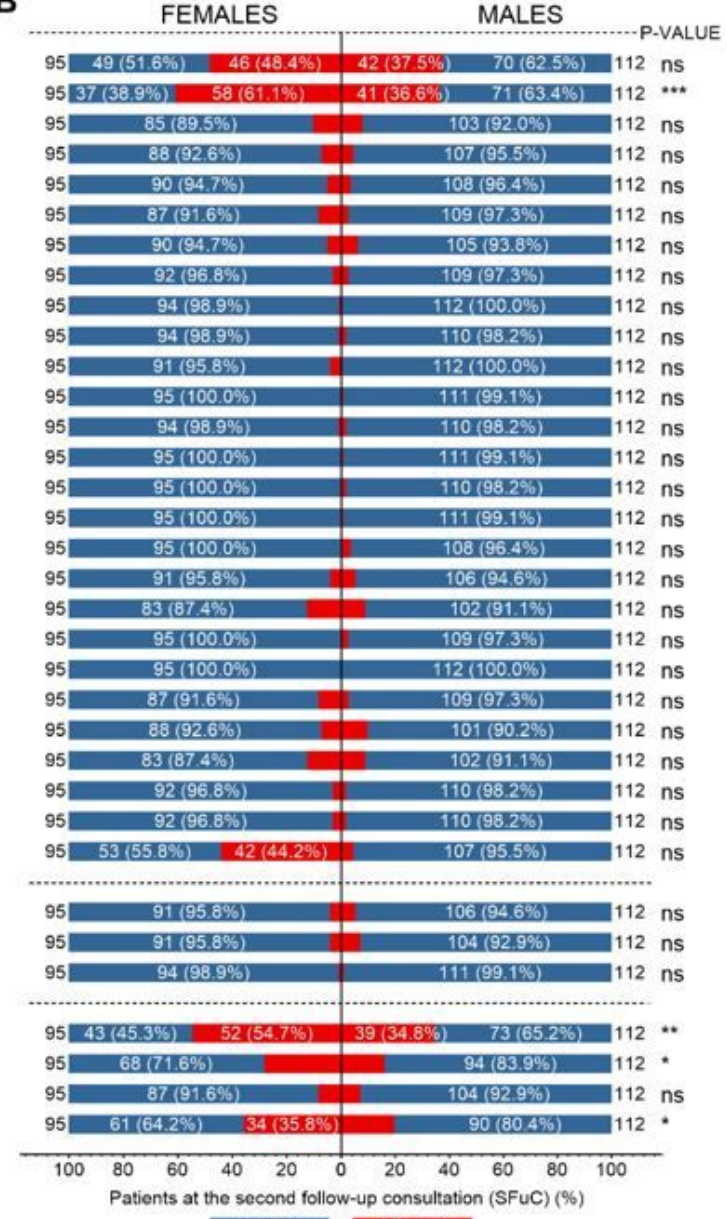

Healny afrected

\section{Figure 2}

Clinical features, physical examination, and mental health of females and males with post-acute sequelae of SARS-CoV-2 infection for the first and second follow-up consultations - 2- and 6-months post symptom onset of COVID-19. Frequencies of symptoms presented in $N^{\circ}$ (\%) of the total for each gender in the FFuC (A) and SFuC (B). 
A

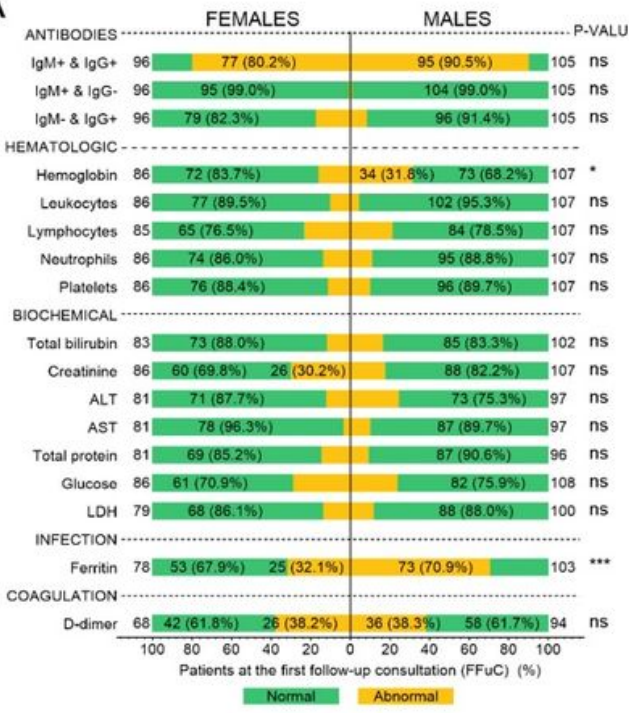

B

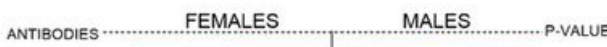

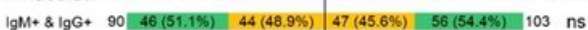

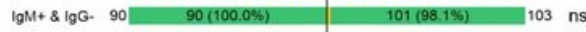

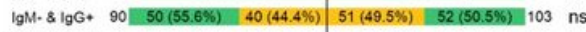
HEMATOLOGIC........

\begin{tabular}{ll|lll} 
Hemoglobin 93 & $84(90.3 \%)$ & $49(47.6 \%)$ & $54(52.4 \%)$ & 103
\end{tabular} \begin{tabular}{l|l|l|l|l|l|} 
Loukocylos 93 & 85(91.45) & $96(93.2 \%)$ \\
\hline
\end{tabular} \begin{tabular}{l|l|l|l|l|l|l|} 
Lymphocytos 93 & $71(76.3 \%)$ & $89(86.4 \%)$ & $103 \mathrm{~ns}$
\end{tabular}

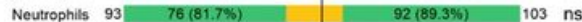

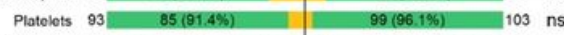
BIOCHEMICAL …

\begin{tabular}{l|l|l} 
Total bilirubin 92 & $84(91.3 \%)$ & $77(78.6 \%)$
\end{tabular}

\begin{tabular}{l|l|l|l|l|l|l|l|l|l|} 
Creatinine 97 & $69(71.15)$ & $93(82.3 \%)$ & 113 & $\mathrm{~ns}$
\end{tabular}

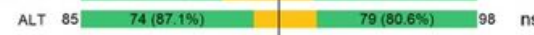
\begin{tabular}{ll|l|ll} 
AST 85 & $79(92.9 \%)$ & $89(90.8 \%)$ & 98 & ns
\end{tabular}

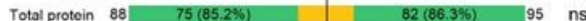
\begin{tabular}{|c|c|c|} 
Glucose 93 & $70(75.3 \%)$ & $83(79.8 \%)$ \\
\hline
\end{tabular}

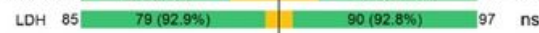

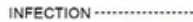

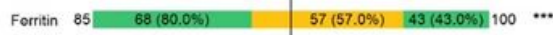
COAGULATION.

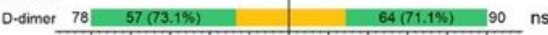

$\begin{array}{lllllllllll}100 & 80 & 60 & 40 & 20 & 0 & 20 & 40 & 60 & 80 & 100\end{array}$

Patients at the second follow-up consultation (SFUC) (\%)

Normal Abnormal

$\mathrm{C}_{\mathrm{H}}$

HEMATOLOGIC Normal(N) Abnormal(Abn) 25\% -75\% I Range within 1.51QR O Mectian

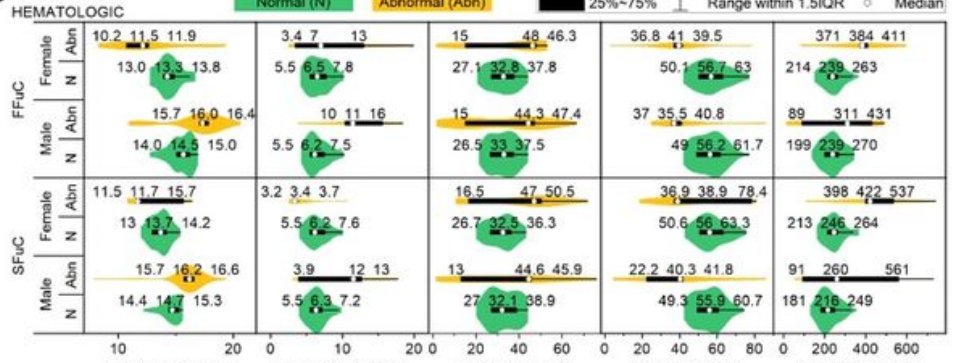

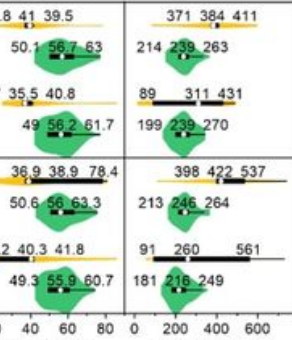

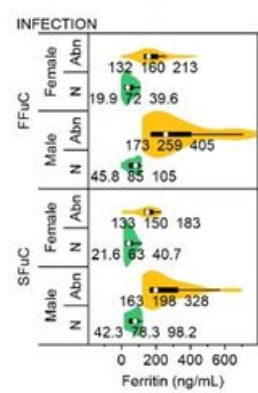

COAGULATION

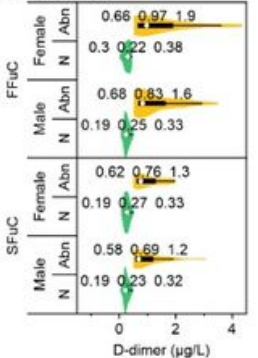

Hemoglobin $(g / \mathrm{d} / \mathrm{f}$
BIOCHEMICAL.

Forritin (ng/mL)

D-dimer ( $\mu g$

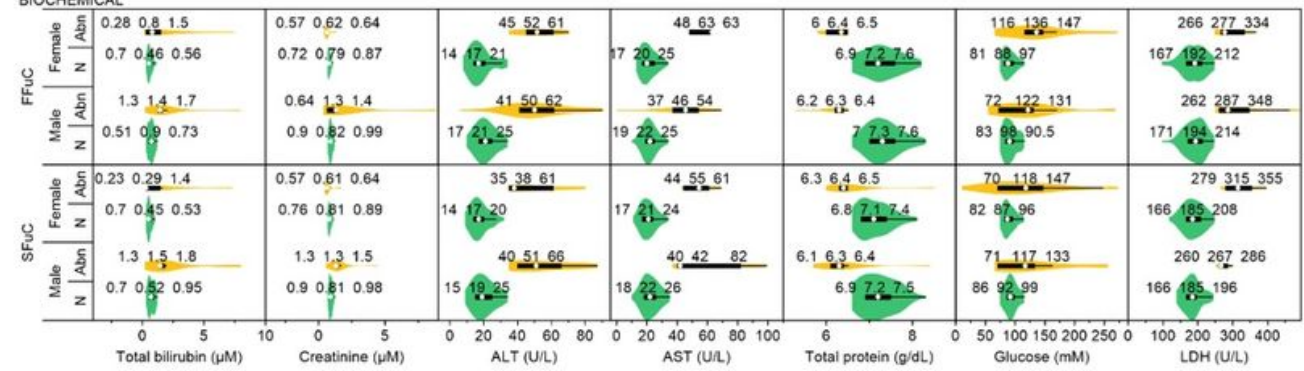

\section{Figure 3}

Laboratory indices of females and males with post-acute sequelae of SARS-CoV-2 infection for first/second follow-up consultations. Frequencies of normal (green color) and abnormal (yellow color) laboratory indices presented in $\mathrm{N}^{\circ}$ (\%) of the total for each gender for the FFuC (A) and SFuC (B). (C) Violin plots show the distribution, median, and quartiles of the laboratory indices for FFuC, SFuC, and gender. 

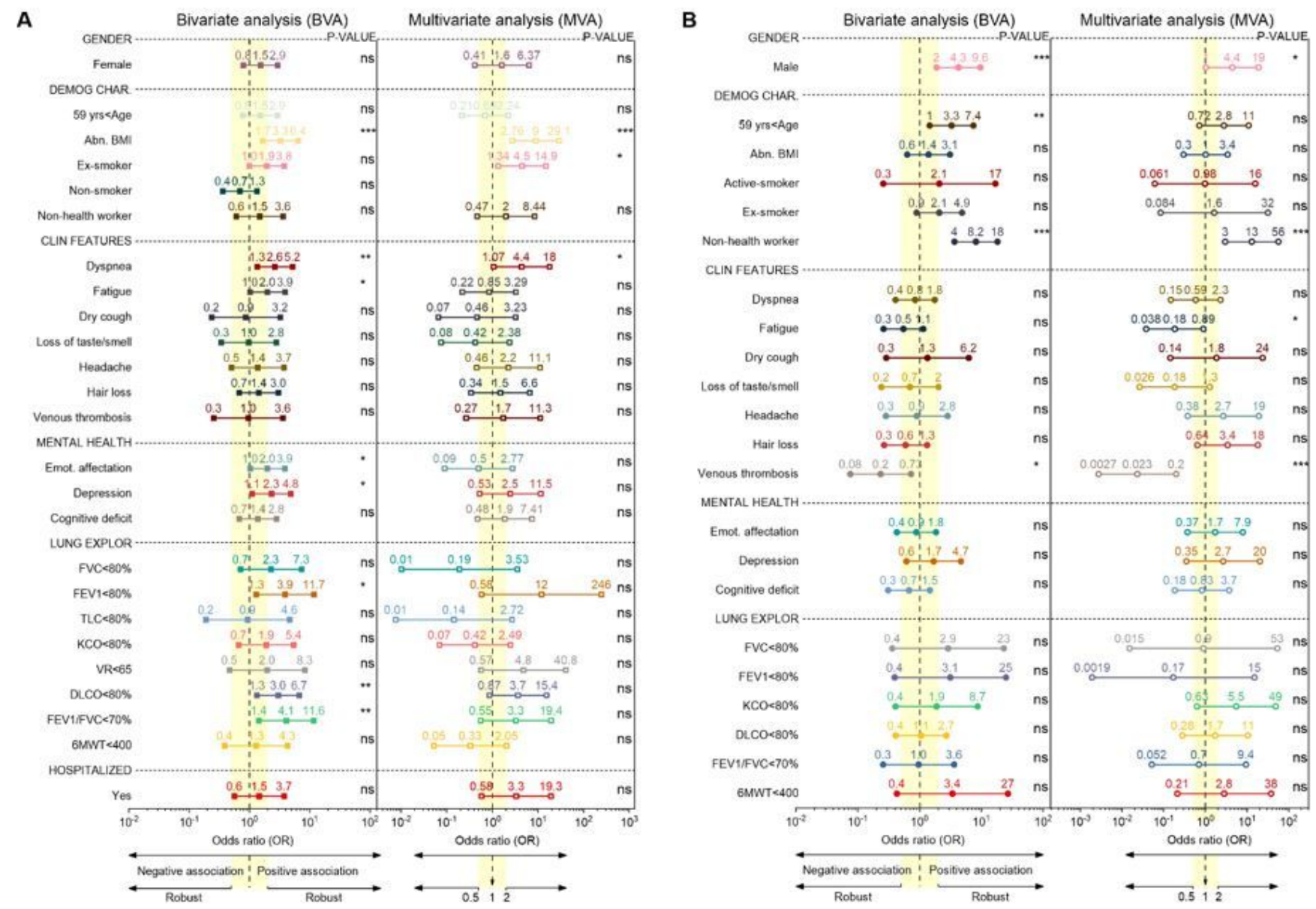

\section{Figure 4}

Forest plot of the odds ratio and its Cls (95\%) values for the bivariate and multivariate analysis. Relationship between the PASC features with the pre-existing respiratory disease (A) and hospitalization (B). The vertical yellow band delimits the regions for a robust ( $O R \leq 0.5$ or $2 \leq 0 R$ ) or weak association $(0.5<0 R<2)$. The $p$-values of the right column indicate the level of significance. 
A

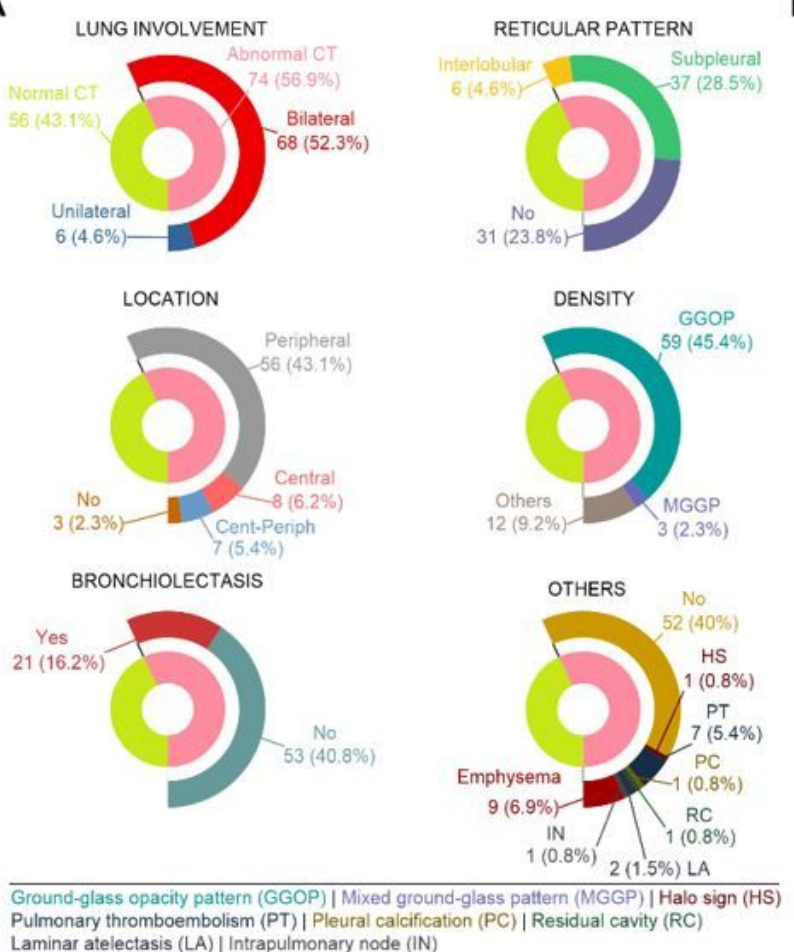

B

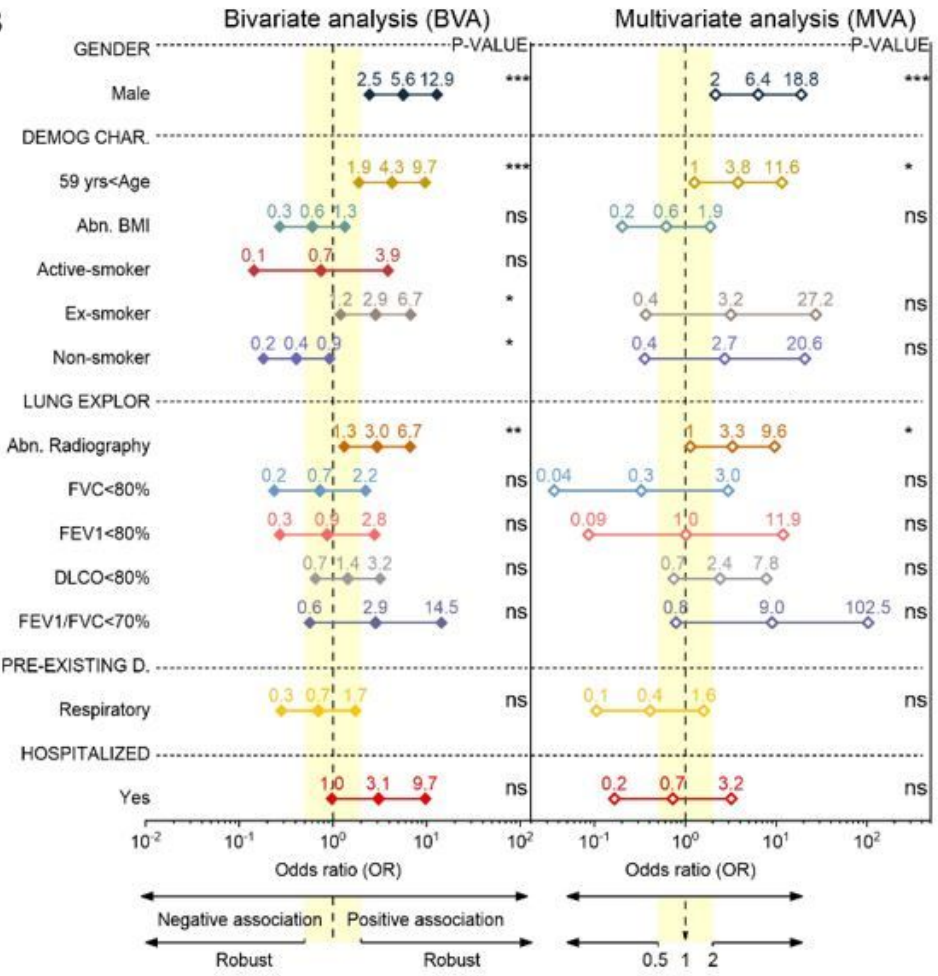

\section{Figure 5}

(A) Chest CT scan findings in all patients at the 6-month follow-up. (B) Forest plot of the odds ratio and its Cls (95\%) values for the bivariate and multivariate analysis. Relationship between the PASC features with the normal/abnormal chest CT outcome. The vertical yellow band delimits the regions for a robust $(\mathrm{OR} \leq 0.5$ or $2 \leq \mathrm{OR})$ or weak association $(0.5<\mathrm{OR}<2)$.

\section{Supplementary Files}

This is a list of supplementary files associated with this preprint. Click to download.

- SingleMultipleLongCovid192021SupplementaryDoc.docx 